\title{
Pedagogic Perspectives on Chinese Characters Teaching for Latin
} \section{American Students}

\author{
Jiajia $\mathrm{Yu}^{1}$, Alexis Lozano ${ }^{2}$ \\ ${ }^{1}$ International School, Jinan University, Guangzhou, China \\ ${ }^{2}$ Department of Chinese, Sun Yat-sen University, Guangzhou, China \\ Correspondence: Alexis Lozano, Department of Chinese, Sun Yat-sen University, Guangzhou, China. Tel: \\ 82-15521182268. Email: alexis.lozano@gmail.com
}

Received: October 11, $2017 \quad$ Accepted: October 26, $2017 \quad$ Online Published: November 28, 2017
doi:10.5539/ass.v13n12p45

\begin{abstract}
Chinese characters are one of the most representative components of Chinese language. However, due to its complexity, the teaching of the language has become an important research topic. With the expansion of this language, it is important to analyze and reconsider approaches to inspire and guide students with different cultural backgrounds, languages and learning habits, and highlight their advantages and disadvantages. Based on two beginner level groups of Chinese in Mexico, this report analyzes teaching strategies, pedagogical activities and students' attitudes towards two professors, a local Mexican teacher and a Chinese teacher. After observing both classes we found significant differences on their approaches to teach Chinese characters. The Chinese teacher emphasized the importance of characters as a communication tool and therefore tried to develop accuracy and efficiency, while the Mexican teacher focused on knowledge about characters, the association with students' own experiences and self-directed learning techniques. We conclude with making remarks about which of these teaching approaches are more suitable for the teaching context of Chinese language in Latin American countries like Mexico.
\end{abstract}

Keywords: Chinese characters, teaching approaches, Chinese teaching in Mexico

\section{Introduction}

The writing system of Chinese characters is one of the most complicated writing systems in the world and probably the richest one in historical and cultural content. It is also a strong impetus for students globally who decide to learn Mandarin Chinese with the goal to come to read and write the language. However, the Chinese writing system is considered the principal challenge for students who have only mastered alphabetic writing systems (Everson, 2011). How to teach Chinese characters has become a major research topic in the teaching of Chinese (Li \& Ruan, 2012), and still remains to be an area of constant debate because of the variety of research results and different analysis perspectives, especially in the case of foreign learners of Chinese.

At present thousands of students are learning Chinese in Latin America. They are attracted to learn this language not only by the rapid development of Chinese economy but the deep interest in learning a language and culture so distinct from their own (An, 2017). Since learning the Chinese writing system constitutes a challenge for both teachers and students, it is important to analyze the teaching efficiency and the way that teachers and students perform and participate in the pedagogic process. This article presents a descriptive analysis of different methods in teaching characters to beginners based on two case studies of Chinese Mandarin courses in Mexico, and discusses different pedagogical approaches that can be implemented during the teaching of Chinese characters in Latin American countries which have an education system and culture different from that of China and other countries such as the United States.

The Chinese writing system is a logographic system that represents a concept corresponding to a word or part of a word through a graphic representation (Huang \& Liao, 2007). In Chinese, a character represents the sound of a syllable. However, it is not strongly associated with the sound of a particular syllable as it is in phonetics-based writing systems. The independence from pronunciation makes it impossible to transcribe spoken language if one does not have the knowledge on how to write each word in its context. This is the main reason for the high difficulty in learning to read and write the characters, but the writing system brings benefits that were of vital significance in history as it creates the possibility to achieve communication between people from different regions where various dialects are spoken, and to attain the understanding of texts in different eras.

Although learning the Chinese writing system is much more complicated than a phonetics-based one, the characters have common characteristics that facilitate comprehension and learning, which are employed by native and non-native learners of Chinese (Shen, 2005). Within a character there are components and radicals appearing in different characters that usually share some aspect in common. It can correspond to similar phonetic aspects or some semantic relation, for example 饭 (fan4) meaning food contains two components with a left-right structure. The left part indicates semantic information as it relates to food and the right part 反 (fan3) meaning inverse is related to the 
pronunciation of the character.

In the case of teaching characters to adults who are accustomed to using an orthographic rather than logographic system, there is a constant debate on how the characters should be taught, including the optimal time for character teaching (Ye, 2013), the efficiency of the use of visual and phonological mnemonics (Kuo \& Hooper, 2004), radical analysis (Taft \& Chung, 1999; Shen, 2007), effectiveness of stroke-order learning (Hsiaung, Chang, Chen, \& Sung, 2017) and the use of electronic resources in character learning (Lam et al., 2001).

Although comparisons of different teaching approaches have been made, results of these researches are only applicable to a few aspects in character teaching. They guide teachers in the effectiveness of different exercises and help teachers to make an informed decision in the design of the teaching plan based on the course objectives and the characteristics of the students. Nevertheless, few research has focused on the macroscopic aspects regarding pedagogic approaches and objectives in the process of character teaching.

This article is based on observing classes of two first-level groups of Chinese language in the Confucius Institute of the National Autonomous University of Mexico (UNAM) and analyzes the process and results of different teaching methods employed by two teachers in order to know how they affect the acquisition of knowledge, obtainment of skills for character recognition and writing, as well as changes in student attitude. The aim of this article is to present the complexity and importance of research on Chinese character teaching and to highlight aspects that should be taken into account when taking an approach to the teaching of this writing system.

\section{Case study}

\subsection{Objects of analysis}

We observed pedagogical activities in two groups of first-level Chinese course from week 2 to week 8 in the spring of 2017 at the Confucius Institute, UNAM. Opinions and comments from students and teachers were collected. During this period, students began to learn characters related to basic daily communication. Both groups used the same textbook Hanyu Jiaocheng (Course on Chinese Language) Vol. 1 Part one, which covers the basics of the language and elementary communicative skills such as shopping and ordering food, and requires students to master about 60 characters. An important aspect to be mentioned of this book is that it does not take into account the difficulty of characters. It is organized based on grammatical concepts and communication needs, so the teachers can modify the teaching sequence to reduce difficulty of the course. Group A had 23 students and was taught by a local Mexican teacher, while group B had 25 students and was taught by a teacher of Chinese nationality. Both teachers have more than 5 years of experience in teaching Chinese.

\subsection{Case description}

Below is a description of highlighted parts of both classes. The contents related directly to the teaching of characters will be analyzed especially in the following aspects.

\subsubsection{Teaching objectives and methods}

Through a brief interview both teachers were asked how they thought the teaching of characters should be and a summary of their answers is given below:

Group A: The teacher believes that the learning process must be as active as possible, trying to arouse the interest and attract the attention of the students so as to allow learning in advanced stages to be simpler and self-directed, and to help students remember and give them a wider context. She mentioned, "I try to relieve stress from the classroom where we can talk freely about the characters so as to make students feel that the Chinese characters are not too difficult, because they were also hard for me when I was a student. In this way I try to give them a message that they can adopt whatever methods that suit them the best to learn characters."

Group B: The teacher bases the class on her knowledge of the language structure and believes that it is important to learn characters in an orderly way and pay attention to details of the characters. She said, "The characters you learn in the first-level period will be the basis for later stages, so it is very important for students at this stage to know well how to write characters, including the basic strokes, stroke order, the radicals and their meaning, and the size and proportion of each component of the characters. If not, it will be difficult to get rid of bad habits later. Also, practicing writing ensures that you learn to read quickly."

\subsubsection{Ways to introduce and teach the characters}

Group A: In the beginning of week 2 the teacher explained the differences between orthographic and logographic writing systems, giving several examples of cultures employing logographic systems, particularly the Maya civilization as reference which developed in Mexico and Central America. The Maya people used glyphs to represent concepts which in turn were related to the sound of a syllable just like Mandarin Chinese. The teacher gave the example of the word montaña (mountain) (Figure 1) to show the similarities in the logic of the characters (a pictorial representation associated with a concept) and that the Chinese writing is not very complex compared to the Maya system, in which each symbol has many more details as can be seen on the character mountain. She also made use of the reference to the Maya writing system to elucidate another similitude between Chinese characters and Mayan hieroglyphics. The use of meaningful components or radicals to form new symbols reduces learning and writing difficulty as shown in Figure 2 where the component of the Mayan word $i k$ ' is inserted at the center of another 
component which represents a day, forming the word "day of wind" in the same way that the mouth radical 口 (kou3) in Chinese can be inserted into the door radical 门 (men2), forming the word 问 (wen4, to ask). After the historical introduction and presenting the meaning of some basic character 人 (people), 山 (mountain), 日 (sun) and 木 (wood) the teacher did not give any direct information on characters but carried out various activities on the interpretation of characters and radicals in which presentation was given by the students.
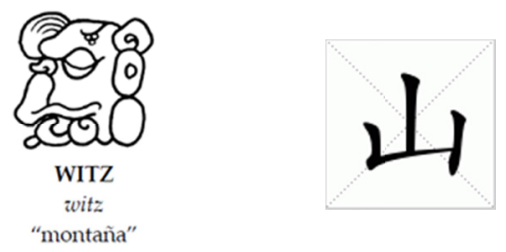

Figure 1. The word mountain in Mayan and Chinese
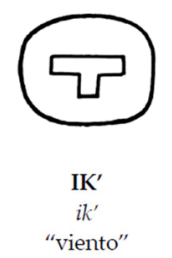

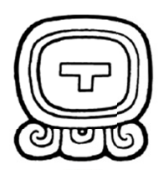

IK'

Signo de día $i k^{\prime}$ ("viento")

Figure 2. The word $i k$ ' forms "day of wind"

Group B: The teacher introduced characters as the only writing system that is valid in China, highlighting its importance with respect to the phonetic transcription pinyin and emphasizing that the characters required attention and mastery of basic strokes and stroke order. The first two weeks stroke exercise was carried out, in which students learned the shape, direction and name of each stroke in written Chinese. In later classes, after orally learning the meaning and use of new words of each lesson, the teacher demonstrated how each character should be written. While the teacher wrote a large-size character on the board she pronounced the name of the radicals appearing in the character to help students recognize the components. She emphasized the proportion, attention to details and the need for practice so as to finally master the characters.

\subsubsection{Class activities}

Group A: Prior to the beginning of each lesson the teacher presented objectives of the lesson and a list of required characters. The characters were divided into several groups and each group was assigned to a student. The teacher asked the students to search in any medium where they had access to an explanation why the character and its components are written that way and present it to their classmates in the next class. In the next class students presented their research results and discussed among them which one was the best. This strategy was repeated three times during the six weeks when the class was observed. The resources used by the students included dictionaries, web pages, Chinese friends and their own imagination.

Another activities that involved the use of characters were a lottery game created by the students (a Mexican traditional game similar to the game Bingo) and the creation of dialogues similar to those in the textbook by writing sentences freely on the board and acting out the created dialogue.

Group B: After introducing each character in the lesson the teacher asked the students to write down the characters in the notebooks following the stroke order, and checked the characters one by one.

One week after the completion of a lesson the teacher gave a dictation of the most important characters and sentences so as to evaluate the students' progress in learning the characters and correct the errors that they might make.

In addition, character and radical recognition games were organized in which the teacher mentioned a word or radical, and the students competed in turns to beat the corresponding characters or radicals on the board, earning points for their team.

\subsubsection{Homework}

Group A: The most frequent homework was described in class activities Group A that students made use of network resources (including videos, forums and online dictionaries) and even used their own imagination to find concepts that helped them associate the characters with their sound or meaning. On other occasions the teacher assigned homework of completing textbook exercises using characters.

Group B: The teacher assigned homework of copying characters and sentences for each lesson.

\subsubsection{Error correction}

Group A: There were no errors in the students' presentation on the meaning of the characters since the key was to find 
a way to remember and associate. Therefore, even if the presented explication did not match the historical origin of the character, it was still instrumental for students to remember and comprehend the logic of the characters, which motivated them to present different interpretations without being afraid of errors, for instance, when studying the character 文 (wen2, culture) students offered several interpretations:

“文 has to do with the first characters that were written in tortoise shells, so the character for writing and culture is represented with a turtle."

"Culture is what gives you identity. It is like a big tattoo that you have written on your chest."

"People who had culture and knew how to write were those with a lot of money and ample clothes like the person that seemed walking in the character wearing a large robe."

Some of the interpretations were based on the students' imagination, which is not necessarily the official origin of the characters, but were accepted as useful in order to understand the characters.

In the exercise of writing sentences on the board the students made multiple mistakes in writing the characters. The teacher asked all students to look for the error and to correct it together.

Group B: All the mistakes on shape, strokes and proportion were pointed out by the teacher, sometimes orally while checking the students' writing in class and sometimes marking the mistakes in the homework or dictation.

\subsection{Assessment results}

All students who regularly attended the class in both groups passed the assessments in the period when the class was observed, though a notable increase of writing speed and improvement of character quality could be seen in group B.

\subsection{Opinions and attitude of students}

Students were asked to write a reflection at the end of week 4 and week 8 about their learning experiences, including their opinions about the teaching methods, how much time they spend in learning Chinese per week, difficulties they encounter, progress they have made and overall attitude towards the course.

At week 4, the reflection showed no big difference between two groups. One of the common difficulties mentioned by students was the characters, but most of them remained positive that they would be able to master them one day. However, at week 8, differences of students' attitude towards their own progress in characters could be noticed between group A and B. Students from group A still demonstrated strong interest and confidence in learning characters since they were accustomed to looking for information themselves while students from group B showed more complaints with 15 out of 25 mentioning that they might not have enough time to practice the characters and doubted whether they could learn to write the characters someday.

\section{Discussion}

The teaching results of both groups can be considered a success because the general objectives of the course were attained and most of the students understood the required knowledge as shown in the assessments and had a positive opinion towards both courses. However, it is easy to observe that there are great differences in the teaching approaches, which are highlighted by the educational customs and are worth discussing.

We do not attempt to point out that the teaching methods of both teachers are representatives of local teachers or teachers of Chinese nationality. The observation allows us to analyze ways that can guide the teaching of Chinese characters and their advantages respectively.

Throughout the courses we can see that two aspects of character were developed: ability to read and write and knowledge about reading and writing. The Chinese teacher in group B put a remarkable emphasis on the development of writing skills, sought to achieve the course objectives stated in terms of language acquisition and did not explicitly show the focus on the development of cross-cultural communication abilities, cultural knowledge or learning techniques, while the Mexican teacher in group A put more attention on knowledge about the characters themselves and the ability to analyze them, taking her own learning experience as a reference.

In terms of foreign language acquisition a clear distinction has been made between language learning and language acquisition (Krashen, 1984), since metalinguistic knowledge is not so useful in developing students' communicative capacities as constant communication (including reception and production) of comprehensible messages in the target language. Although acquisition of the writing system follows very distinct mechanisms from those of spoken language, this communicative approach can be effective in reading and writing for several reasons. The memorization and recognition of characters is a process that requires constant practice. The brain should be able to access information on the shape of the characters and the procedure to write them at a relatively high speed. This process can be trained by means of continuous writing, like the teacher did in group B through repetition tasks and by writing characters on the board slowly while pronouncing the sound of the character with the students following her. In this way, students tried to reinforce the connection between the movements, the image of the character and its sound or meaning in their brains by means of Hebbian learning, in which signals that are triggered simultaneously or sequentially in the brain are reinforced, increasing the possibility to repeat the signal sequence in the future.

This kind of training was completed during the learning of some characters in group B, where the learning of characters managed to keep pace with that of spoken language, and characters were written with great care in aesthetic 
aspects and with higher speed. However, a problem emerged as students progressed in the study of the language as can be seen in group B students' reflection at the end of week 8: $60 \%$ of the students mentioned the problem of study time, saying that they probably would not have enough time for practice in the future.

The time problem in the study of characters is a fundamental problem and there have been experts who for this reason consider that the ability of handwriting is not imperative in the study of Chinese and Japanese because of the time it takes and the electronic tools that are currently available (Allen, 2008). It is a principal problem in the repetition-based teaching techniques as those analyzed in group B. Although the pace of progress in the spoken language of the first level is slow owing to the study of pronunciation and basic grammars, the students feel that they do not have sufficient time even to only memorize the most used characters. This strongly discourages students who have learned Chinese for only two months as can be seen in their comments.

The teacher of group A focused more on learning knowledge about writing and acquiring skills to learn and recognize characters. Although it is clear that the main purpose of this knowledge is to obtain writing skills, the teacher did not spend too much time in or out of class on the practice of characters. However, the students achieved similar results to those in group B. There may be several reasons. First, the students are adults who know the way they learn effectively. Second, they have the ability to copy characters or make strokes since everyone knows how to write in Spanish. The training in stroke direction or copying characters may be more applicable to children who do not yet master the use of a pencil, but for adults who have limited time and know how to write, it does not necessarily need to spend so much time on this kind of practice or orientation.

The knowledge imparted by the Mexican teacher did not directly help the students understand more words but arouse their interest and intention in details, linking characteristics of the characters to something with which the students are more familiar such as the Maya writing that is more close to students' life since it was introduced in primary education in Mexico. In addition, the rest of the information concerning characters were presented and discussed by the students themselves, making the class an active place of conversation where students could discuss history, their problem in searching information and even joke with one another. It helps to reduce classroom stress and is beneficial for language acquisition in both oral skills (Philips, 1992) and writing skills (Rai, Loschky, Harris, Peck, \& Cook, 2010).

The use of additional resources in group A encouraged students to be independent in their learning, which is conducive in language learning since the amount of information and skills to learn is high and one cannot depend totally on teachers, particularly in the teaching of Chinese in Mexico where class time is limited and class size is big, making it difficult for teachers to give individual attention.

The discussion among peers and use of their own resources are methods that accord with constructivist principles of education which have demonstrated efficiency in knowledge transfer and motivation (Schunk, 2012). They are fundamental in character learning that requires analytical skills and a longer learning time than many other writing systems even if students only try to obtain reading skills. In this way the teacher of group A played the role of facilitator, orientating discussions while the students helped each other to understand the difficult parts of characters, creating a scaffolding structure to achieve utmost learning, according to Vygotskyan theories of social learning (1978).

Another factor that affects the motivation and perception of the difficulty of characters for the students lies in the pressure to master a correct form of writing. The teacher in group B paid great attention to see if the characters were as correct as possible, directing students' attention to even aesthetic details of the characters. In addition, she exerted pressure on the weekly dictation, in which students always scored low. The dictation and continuous evaluation of results are a highly efficient method to consolidate concepts and abilities in long term memory, surpassing the efficiency of elaboration on concepts in many other cases (Karpicke, 2011). However, this efficiency can only be achieved when the students have already had the knowledge and comprehend it in an efficient manner. In the dictation of group B, many students did not have sufficient time to learn, resulting in bewilderment in their pace of progress. In group A, character exercises were carried out in teams and errors were marked by students themselves without receiving a score, which avoids students from perceiving a high difficulty in the learning process or hurting their self-esteem.

In general the differences between both groups lie in how the teacher perceives the learning of characters based on their own experiences. The Chinese teacher learned the writing system when she was a child and has the knowledge of the language, culture and basic concepts of writing. The reading and writing skills allow her to transcribe what she means to communicate, which takes a long time to obtain. The Mexican teacher has studied a long time in Mexico, learning the spoken language, writing and culture at the same time, thus she believes that students do not necessarily have to spend all their time on practice this ability, and takes practicing characters as an opportunity to relax the class and talk about history and interact with students, which was not the focus of group B. Follow-up research on the use of these two approaches will be useful to identify students' learning outcomes.

\section{Conclusions}

The teaching of Chinese writing system can be developed in different ways. However, the system is not only an auxiliary of the language. If it were only a transcription tool, it might have been abandoned a long time ago, since it is a difficult system and takes time to master as can be noticed when students do repetition exercises. The Chinese characters are a fundamental element that gives identity to the language and constitutes a great part of the Asian culture. 
Therefore, learning the language is not merely a process of memorization and automation in the process of recognizing and writing the necessary symbols for the purpose of communication. Because of its logographic nature, each symbol and its components can be directly related to concepts at a sub-morphemic level that is not necessarily associated with a sound. The concepts reduce the difficulty in the learning process and help students to understand important elements of Chinese culture and history which in turn can become an additional tool for memorization and creativity in a practical way.

The acquisition of this knowledge in the cases analyzed in this article not only helped to arouse students' personal interest in knowing more of this writing system, but was also conducive to reduce the complexity perceived by the students in the writing process. In Latin American countries like Mexico where students usually take a great interest in active class and attend Chinese classes out of interest in learning a new culture, an active learning approach where students learn and comprehend to seek connections with their own knowledge and resources may be more efficient and attractive in the long run.

\section{References}

Allen, J. R. (2008). Why learning to write Chinese is a waste of time: A modest proposal. Foreign Language Annals, 41(2), 237-251. http://www.doi.org/10.1111/j.1944-9720.2008.tb03291.x

AN, X. Z. (2017, May 12). El avance del chino en América Latina. China Hoy. Retrieved from http://www.chinatoday.mx/cul/ChCul/content/2017-05/12/content_740723.htm

Everson, M. E. Best practices in teaching logographic and non-Roman writing systems to L2 learners. Annual Review of Applied Linguistics, 31(2011), 249-274. http://doi.org/10.1017/S0267190511000171

Hsiung, H. Y., Chang, Y. L., Chen, H. C., \& Sung, Y. T. (2017). Effect of stroke-order learning and handwriting exercises on recognizing and writing Chinese characters by Chinese as a foreign language learners. Computers in Human Behavior, 74, 303-310. https://doi.org/10.1016/j.chb.2017.04.022

Huang B. R., \& Liao X. D. (2007). Xiandai Hanyu (zengding si ban, xiace) (Modern Chinese (Revised $2^{\text {nd }}$ ed., Second Volume). Beijing: Higher Education Press.

Karpicke, J. D., \& Blunt, J. R. (2011). Retrieval practice produces more learning than elaborative studying with concept mapping. Science, 331(6018), 772-775. https://doi.org/0.1126/science.1199327

Krashen, S. D. (1987). Principles and practice in second language acquisition. New York: Pergamon Press Inc.

Kuo, M. L. A., \& Hooper, S. (2004). The effects of visual and verbal coding mnemonics on learning Chinese characters in computer-based instruction. Educational Technology Research and Development, 52(3), 23-34. https://doi.org/10.1007/BF02504673

Lam, H. C., Ki, W. W., Law, N., Chung, A. L. S., Ko, P. Y., Ho, A. H. S., \& Pun, S. W. (2001). Designing CALL for learning Chinese characters. Journal of Computer Assisted Learning, 17(1), 115-128. http://www.doi.org/10.1111/j.1365-2729.2001.00164.x

Li, Q., \& Ruan C. (2012). "Hanzi Nanxue" zhi jiaoxue duice (Teaching strategies for the "Chinese characters are hard to learn” problem). 《汉语学习》, (4), 83-90.

Phillips, E. M. (1992). The effects of language anxiety on students' oral test performance and attitudes. The Modern Language Journal, 76, 14-26. https://doi.org/10.1111/j.1540-4781.1992.tb02573.x

Rai, M. K., Loschky, L. C., Harris, R. J., Peck, N. R., \& Cook, L. G. (2011). Effects of stress and working memory capacity on foreign language readers' inferential processing during comprehension. Language Learning, 61, 187-218. https://doi.org/10.1111/j.1467-9922.2010.00592.x

Schunk, D. H. (2012). Learning Theories (6th ed.). New Jersey: Prentice Hall Inc. 2012.

Shen, H. H. (2005). An investigation of Chinese-character learning strategies among non-native speakers of Chinese. System, 33(1), 49-68. https://doi.org/10.1016/j.system.2004.11.001

Shen, H. H., \& Ke, C. (2007). Radical awareness and word acquisition among nonnative learners of Chinese. The Modern Language Journal, 91(1), 97-111. https://doi.org/10.1111/j.1540-4781.2007.00511.x

Schunk, D. H. (2012). Learning Theories (6th ed.). New Jersey: Prentice Hall Inc.

Vygotsky, L. S. (1980). Mind in society: The development of higher psychological processes. London/ Massachusetts: Harvard University Press.

Ye, L. (2013). Shall we delay teaching characters in teaching Chinese as a foreign language?. Foreign Language Annals, 46(4), 610-627. https://doi.org/10.1111/flan.12049

\section{Copyrights}

Copyright for this article is retained by the author(s), with first publication rights granted to the journal.

This is an open-access article distributed under the terms and conditions of the Creative Commons Attribution license (http://creativecommons.org/licenses/by/4.0/). 